\title{
Estudio in vivo del efecto angiogénico de inhibidor de fosfodiesterasa para aplicación posterior en injerto dermoepidérmico*
}

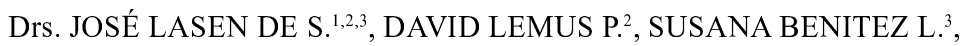 \\ CARLOS SCIARAFFIA M. ${ }^{3}$, SERGIO SEPÚLVEDA ${ }^{3}$, JAIME JANS J. ${ }^{3}$ \\ 1 Programa de Magíster en Ciencias Biomédicas mención Fisiopatología, Escuela de Postgrado, \\ Facultad de Medicina Universidad de Chile. \\ 2 Departamento de Anatomía y Biología del Desarrollo, ICBM, Facultad de Medicina Universidad de Chile. \\ 3 Departamento de Cirugía Hospital Clínico de la Universidad de Chile. \\ Santiago, Chile.
}

\begin{abstract}
\section{Angiogenic effect of sildenafil in a chicken egg model}

Background: Angiogenesis is a complex process. Phosphodiesterase inhibitors may have a direct angiogenic effect. Aim: To determine if phosphodiesterase inhibitors have angiogenic properties, using a chicken egg model. Material and Methods: We used 44 fertilized chicken eggs. A methylcellulose filter was placed over their allantocorionic membrane. This preparation was instilled with different solutions. Group A (Control) received $30 \mu \mathrm{l}$ of saline solution, Group B, C and D received $30 \mu \mathrm{l}$ of a solution made of saline solution and sildenafil at different concentrations of $0.33,1$ and $3.3 \mu \mathrm{g} / \mu \mathrm{l}$, respectively. At day 12 the filters were removed, prepared for histologic analysis, and the number of capillaries in an area of $2250 \mu \mathrm{m}^{2}$ were blindly counted. Statistical analysis was made using variance analysis (ANOVA) with Bonferroni technique $(\mathrm{p}<0.001)$. Results: The number of capillaries counted, per $2250 \mu \mathrm{m}^{2}$, in Groups A, B, C, and D were 11.1 $\pm 0.5,15.4 \pm 1.2,16.6 \pm 0.8$ and $19.2 \pm 0.9$, respectively. The number of capillaries of groups B, C and D were significantly higher than those of group A (control). Moreover, there was a linear relationship between the number of capillaries and sildenafil dose $(\mathrm{p}<0.001)$. Conclusions: In this experiment, sildenafil had a potent angiogenic effect.
\end{abstract}

Key words: Sildefanil, angiogenesis, phosphodiesterase inhibitors.

\section{Resumen}

Introducción: El proceso de angiogénesis es un proceso complejo. El uso de factores proangiogénicos está bien establecido. En este estudio se trató de averiguar si los inhibidores de fosfodiesterasa, además de su rol vasodilatador, tendrían un efecto angiogénico en los tejidos para evaluar su potencial uso terapéutico futuro en injerto dermoepidérmico. Hipótesis: Se plantea como hipótesis que el inhibidor de fosfodiesterasa tiene un efecto angiogénico directo. Material y Método: Se utilizaron 44 huevos de pollo fecundados obtenidos

* Recibido el 17 de Junio de 2010 y aceptado para publicación el 29 de Septiembre de 2010.

Correspondencia: Dr. José Lasen de S.

Santos Dumont 999, Santiago, Chile.

joselasen@gmail.com 
del Instituto de Salud Pública (ISP), a los cuales se les implantó un disco de metilcelulosa sobre la membrana alantocoriónica, a los que luego se les instiló distintas soluciones: Grupo A control, se instiló 30 $\mu 1$ de solución fisiológica. Grupo B, C y D 30 $\mu \mathrm{l}$ de solución con Citrato de Sildenafil a una concentración de $0,33 \mu \mathrm{g} / \mu \mathrm{l}, 1$ $\mu \mathrm{g} / \mu \mathrm{l}$ y $3,3 \mu \mathrm{g} / \mu \mathrm{l}$ respectivamente. Al día 12 se removieron los discos y se fijaron para análisis histológico y se contaron de manera ciega los capilares en área de $2.250 \mu \mathrm{m}^{2}$. Análisis estadístico con método de análisis de varianza (ANOVA) con técnica de Bonferroni $(\mathrm{p}<0,001)$. Resultados: En Grupo A control, existía un promedio de 11,09 capilares/2.250 $\mu \mathrm{m}^{2}$ DS 0,52 . Grupo B 15,35 capilares/2.250 $\mu \mathrm{m}^{2}$ DS 1,19 . Grupo C 16,62 capilares/2.250 $\mu^{2}$ DS 0,82. Grupo D 19,2 capilares/2.250 $\mu^{2}$ DS 0,89 . Se encontró que el numero de capilares era significativamente mayor en los Grupos B, C y D en relación a control $(\mathrm{p}<0,001)$. Además se observó diferencias estadísticamente significativas entre todos los grupos que recibieron tratamiento con dosis progresivas del Citrato de Sildenafil $(\mathrm{p}<0,001)$. Conclusión: Se observó un efecto angiogénico del inhibidor de fosfodiesterasa utilizado, lo que podría ser aplicado en modelo para estudiar angiogénesis en injertos.

Palabras clave: Sildenafil, angiogénesis.

\section{Introducción}

La angiogénesis se puede definir como la formación de vasos sanguíneos nuevos a partir de vasos preexistentes, a diferencia de la neoangiogénesis en que se forman a partir de células totipotenciales en el período embriológico ${ }^{1}$. La angiogénesis ocurre tanto en el período embriológico como también en distintos momentos de la vida, siendo un proceso complejo que participa tanto en procesos patológicos como también en múltiples procesos fisiológicos beneficiosos como son, la reparación de los tejidos, embriogénesis, ciclo menstrual, entre otros ${ }^{2}$.

Normalmente, las células endoteliales (CE) maduras, se encuentran en etapa mitótica Go (Etapa de reposo proliferativo), siendo una población estable que no prolifera bajo condiciones fisiológicas. A pesar de esto, frente a situaciones de estimulación de la angiogénesis, las CE entran en fase de proliferación activa durante los procesos de reparación fisiológica y patológica ${ }^{3,4}$. Así, el número y densidad de neocapilares formados en estos procesos guarda directa relación con el eventual éxito de la respuesta fisiológica o patológica que requirió de esta reactivación de las CE, como se puede ver por ejemplo en la sobrevida de los injertos dermoepidérmicos ${ }^{5,6}$. Todo esto ha generado un masivo interés por estudiar el complejo fenómeno de la angiogénesis, entenderlo y particularmente buscar la manera de regularlo a través de sustancias que modifiquen, estimulen o inhiban a los factores involucrados en este proceso.

La angiogénesis es un proceso altamente regulado donde participan un gran número de factores proangiogénicos como antiangiogénicos, dentro de los que podemos nombrar a la angiostatina, VEGF, entre otros ${ }^{7}$. La mayoría de los estudios acerca de sustancias proangiogénicas se basan en factores de crecimiento conocidos como VEGF y FGb. Últimamente, han aparecido estudios de sustancias que en un principio no se les asignó un rol en el proceso angiogénico, como por ejemplo el Óxido Nítrico
$(\mathrm{NO})^{8,9}$. De hecho, existen escasos reportes acerca de su rol en la angiogénesis, enfocándose la mayoría de los estudios en el efecto vasodilatador, por estimulación de la generación de cGMP en células musculares lisas (CML) vasculares ${ }^{10,11}$.

Como se comentó anteriormente han aparecido escasas investigaciones que evalúan el rol proangiogénico del NO, observándose en todos ellos una estimulación de la proliferación endotelial y angiogénesis mediada por $\mathrm{VEGF}^{12-14}$. El rol del $\mathrm{NO}$ en el proceso angiogénico se ha visto que tiene su génesis en el aumento del cGMP, el cual estimularía la vía angiogénica del VEGF que actuaría en el promotor de migración y proliferación de las células endoteliales que se ensamblan en estructuras tubulares para canalizar la sangre ${ }^{15,16}$. Algunos de estos estudios han observado que en condiciones donde se incuban células musculares lisas junto con $\mathrm{NO}$, los niveles de VEGF se incrementan de manera directamente proporcional y que con la administración de un antagonista de la Oxido Nítrico Sintasa (NOS) ocurría lo contrario ${ }^{17,18}$.

Dentro de los factores involucrados en el proceso de angiogénesis mediada por el NO se ha puesto énfasis en la regulación de una familia de enzimas conocida como Fosfodiesterasa (PDE). Existen múltiples tipos de PDE, con distintas funciones. Respecto a las PDE implicadas en los procesos de angiogénesis se ha observado que la PDE1, PDE2, PDE3, PDE4, PDE5 y PDE7 son expresadas en CE afectando la migración, proliferación y apoptosis de estas ${ }^{19-21}$.

Por otro lado tenemos a las ciclasas, que son enzimas implicadas en la generación de nucleótidos cíclicos como son la guanilil ciclasa (GC) y la adenil ciclasa (AC). Ambas enzimas aumentan los niveles intracelulares de cGMP y cAMP, respectivamente. La GC soluble activada por NO ha sido ampliamente estudiada principalmente en su rol en la relajación de las CML vasculares, pero que últimamente se ha observado tendría efectos positivos en la migración, 
proliferación, crecimiento y organización de capilares en estudios in vitro y angiogénesis in vivo ${ }^{11,22,23}$.

El Citrato de Sildenafil (SDF) se ha usado ampliamente como dilatador de las arterias penianas en pacientes con disfunción eréctil ${ }^{24}$. EL SDF es un inhibidor selectivo de la PDE5 cGMP específica, la cual cataliza la hidrólisis del cGMP. La inhibición de la PDE5 aumentaría los niveles de cGMP intracelular lo que llevaría a la relajación de las células musculares lisas vasculares (CML) ${ }^{25}$.

El SDF es un potente inhibidor de PDE5 (IC50 de 3,9 $\mathrm{nM}$ ), con una alta selectividad por PDE5 ( $>$ 1.000 -fold) sobre PDE2, PDE3 ${ }^{8,26}$. De acuerdo al Sistema de Clasificación Biofarmacológica de la FDA, el SDF es un fármaco tipo 1, que lo caracteriza por su alta solubilidad, de $3,5 \mathrm{mg} / \mathrm{ml}$, y permeabilidad. La dosis comúnmente usada de SDF para el tratamiento de la impotencia funcional va de $1 \mathrm{mg} /$ $\mathrm{kg}$ a $\operatorname{los} 1,5 \mathrm{mg} / \mathrm{kg}$, mientras que su uso en hipertensión pulmonar en niños es mayor usándose de $2 \mathrm{mg} /$ $\mathrm{kg}$ hasta los $10 \mathrm{mg} / \mathrm{kg}^{16}$. Existen estudios que han demostrado un aumento además de la angiogénesis, sinaptogénesis y neurogénesis usando dosis de 10 $\mathrm{mg} / \mathrm{kg}$ de SDF, lo que no se ha observado con dosis menores $^{16}$.

Se plantea como hipótesis que la aplicación local de Citrato de Sildenafil tendría un efecto proangiogénico en la membrana alantocoriónica de pollo (MAC). Además nos planteamos como objetivos establecer un modelo reproducible de neoangiogénesis en MAC mediante la implantación de un filtro de metilcelulosa y medición de capilares. Finalmente como objetivo nos propusimos evaluar el potencial angiogénico del SDF para su eventual uso en experimentos usando injertos dermoepidérmicos.

\section{Material y Método}

Incubación de 44 huevos fecundados de gallina tipo White Leghorn con un peso promedio de $20 \mathrm{gr}$, adquiridos del Instituto de Salud Pública, en cámara a $37^{\circ} \mathrm{C}$ con humedad controlada. La incubación se realizó en posición horizontal, marcándose en su superficie superior con una cruz para poseer una referencia con el embrión.

A las 48 hrs se realizó un orificio en el polo agudo del huevo, mediante el cual se extrajeron $2 \mathrm{ml}$ de albúmina mediante pipeta Pasteur, graduada y acodada, lo que permitiría el descenso de la yema, impidiendo así la adherencia del MAC con la tectácea de la cáscara. Posteriormente se sella el orificio con cinta adhesiva.

Luego se coloca cinta adhesiva en la zona marcada con la cruz, para después, mediante tijera fina esterilizada, realizar una ventana de aproximada- mente $2 \times 3 \mathrm{~cm}$, la cual se sella con cinta adhesiva. Los huevos continúan su desarrollo en la cámara de cultivo.

Al día $9^{\circ}$ de incubación, se realiza el desecho de aquellos huevos con embriones muertos, para luego aleatorizar los restantes con modelo de computación SPSS, separándolos en 4 grupos, a los cuales se les retira la cinta adhesiva y se deposita el filtro de metilcelulosa ( $1 \mathrm{~cm}$ de diámetro, grosor $150 \mathrm{um}, 0,25$ um poro) instilándose con micropipeta $30 \mathrm{uL}$, por huevo, de las distintas soluciones sobre el filtro, las cuales dan como resultado 4 grupos:

1. Grupo A o Control $(n=9)$ donde se instilan $30 \mathrm{uL}$ de solución fisiológica estéril

2. Grupo B $(n=9)$ donde se instilan $30 \mathrm{uL}$ de solución fisiológica con 10 ug de Citrato de Sildenafil (SDF) que corresponde a una concentración de $0,33 \mathrm{ug} / \mathrm{uL}$ o una dosis correspondiente de $1 \mathrm{mg} / \mathrm{kg}$.

3. Grupo $C(n=9)$ donde se instilan $30 \mathrm{uL}$ de solución fisiológica con 30 ug de Citrato de Sildenafil (SDF) que corresponde a una concentración de $\mathrm{lug} / \mathrm{uL}$ o una dosis correspondiente de $3 \mathrm{mg} / \mathrm{kg}$.

4. Grupo $\mathrm{D}(\mathrm{n}=9)$ donde se instilan $30 \mathrm{uL}$ de solución fisiológica con 100 ug de Citrato de Sildenafil (SDF) que corresponde a una concentración de 3,3 $\mathrm{ug} / \mathrm{uL}$ o una dosis correspondiente de $10 \mathrm{mg} / \mathrm{kg}$.

Al $12^{\circ}$ día (Figura 1 ) se extraen los filtros de metilcelulosa con tijera estéril junto con la MAC adherido a estos, para luego realizar la preparación histológica con tinción de Hematoxilina Eosina. Finalmente, se realizó recuento de manera ciega, cubriendo con cinta adhesiva los rótulos de grupo, de los capilares en los cortes histológicos usando microscopia óptica con aumento de $40 \mathrm{x}$, empleando un retículo de $0,5 \mathrm{~cm}^{2}$ dividido en 10 lo que da un área de análisis de $2.250 \mu \mathrm{m}^{2}$. En cada preparado se realizó 1 conteo en cada corte histológico, lo que da como resultado 5 conteos por preparado.

La significación estadística de la comparación de los distintos grupos se determinó mediante análisis de varianza (ANOVA) utilizando el test de Bonferroni para comparaciones múltiples (Método Paramétrico).

Por otro lado, nos propusimos identificar la concentración angiogénica mínima (CAM) del SDF utilizando un modelo de Regresión Lineal, para determinar la menor concentración del SDF capaz de promover una angiogénesis significativamente mayor a la angiogénesis inflamatorio del grupo control.

\section{Resultados}

De 44 huevos fecundados, murieron 8 durante los primeros 9 días de incubación, por lo que quedaron 36 huevos viables, los que fueron aleatorizados en 4 


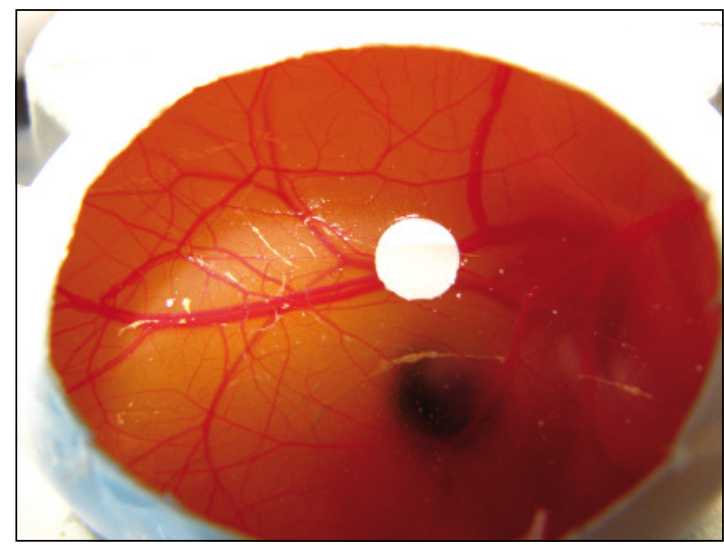

Figura 1. Huevo fecundado mostrando la MAC $12^{\circ}$ día de incubación con el filtro de metilcelulosa.

grupos de 9 huevos cada uno, a cada uno de ellos se les instiló $30 \mathrm{uL}$ de solución fisiológica (SF) con o sin SDF a distintas dosis (Tabla 1).

Se realizaron 5 conteos en cada huevo lo que dió como resultado 45 conteos por grupo analizado. El conteo promedio de capilares de la MAC en los distintos grupos puede ser observado en el Figura 2. Se puede observar que el grupo control, el cual recibió únicamente $30 \mathrm{uL}$ de $\mathrm{SF}$, fue de $11,16 \pm 1,28$ cap $/ 2.250 \mu \mathrm{m}^{2}$. Respecto al recuento de los capilares en los grupos que recibieron tratamiento con SDF se pudo observar que en el:

1. Grupo B, que recibió una dosis de $1 \mathrm{mg} / \mathrm{kg}$ de SDF, el conteo de vasos promedio fue de 13,67 \pm $1,57 \mathrm{c} \mathrm{cap} / 2.250 \mathrm{um}^{2}$.

2. Grupo C, que recibió una dosis de $3 \mathrm{mg} / \mathrm{kg}$ de SDF, el conteo de vasos promedio fue de 15,96 \pm $1,38 \mathrm{cap} / 2.250 \mathrm{um}^{2}$.

3. Grupo D, que recibió una dosis de $10 \mathrm{mg} / \mathrm{kg}$ de SDF, el conteo de vasos promedio fue de $19,33 \pm 1,65 \mathrm{cap} / 2.250 \mu \mathrm{m}^{2}$ (Figura 3).

Al realizar el análisis de varianza ANOVA con el método de Bonferroni, (Tabla 2), se pudo observar que existían diferencias estadísticamente significativas entre el grupo control y los que recibieron tratamiento ( $\mathrm{p}$ value $<0,001$ ). Además, y utilizando el mismo método de análisis estadístico, se pudo observar que también existían diferencias estadísticamente significativas entre los grupos que recibieron tratamiento ( $\mathrm{p}$ value $<0,001$ ). Para buscar la CAM se utilizó como método una Regresión Lineal, que permitió determinar que el número de vasos contabilizados que no sea significativo es de 12,47 cap $/ 2.250 \mu \mathrm{m}^{2}$, valor que permite calcular una CAM del SDF de $0,16 \mathrm{ug} / \mathrm{uL}$, la cual corresponde a una dosis de $0,24 \mathrm{mg} / \mathrm{kg}$ (Figura 4).

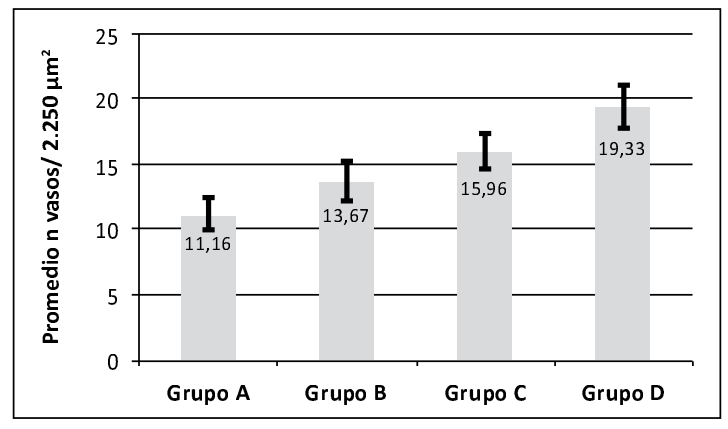

Figura 2. Contabilización de capilares en distintos grupos tratados con SDF.

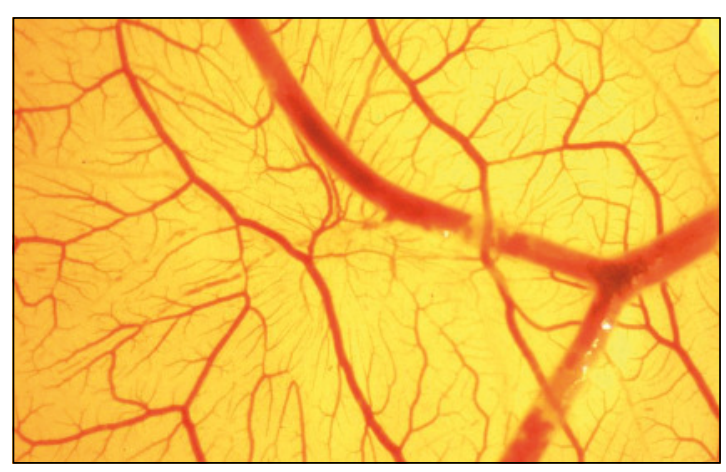

Figura 3. Fotografía macroscópica de vasos MAC en grupo D con SDF $10 \mathrm{mg} / \mathrm{kg}$.

Tabla 1. Concentración y Dosis de SDF aplicada en grupos

\begin{tabular}{lcccc}
\hline & \multicolumn{4}{c}{ Grupos } \\
& A & B & C & D \\
\hline Concentración (ug/ul) & Control & 0,33 & 1 & 3,3 \\
Dosis (mg/kg) & Control & 1 & 3 & 10 \\
\hline
\end{tabular}

Tabla 2. Análisis de Varianza (ANOVA) según método Paramétrico de Bonferroni

\begin{tabular}{ccccc}
\hline \multicolumn{5}{c}{ Grupos } \\
Grupos & A & B & C & p value \\
\hline \multirow{2}{*}{ B } & 2,51111 & & & \\
& 0,000 & & & $\mathrm{p}<0,001$ \\
C & 4,8 & 2,28889 & & \\
& 0,000 & 0,000 & & $\mathrm{p}<0,001$ \\
D & 8,17778 & 5,66667 & 3,37778 & \\
& 0,000 & 0,000 & 0,000 & $\mathrm{p}<0,001$ \\
\hline
\end{tabular}

$(\mathrm{p}$ value $<0,001)$ 


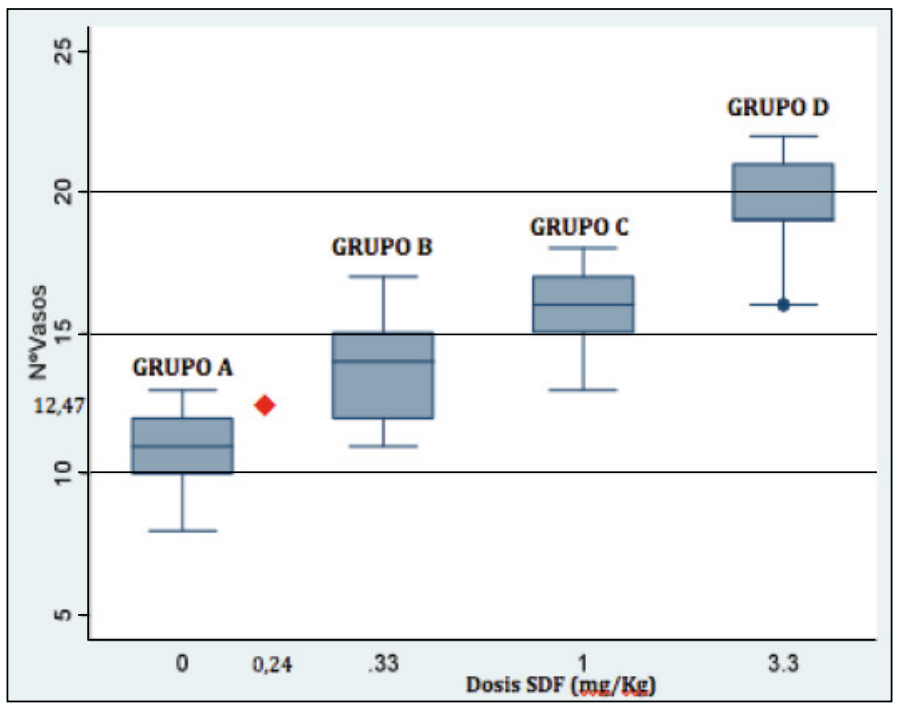

Figura 4. Regresión Lineal Múltiple para identificar la CAM del SDF. • Número de vasos por campo donde $\mathrm{p}=$ NS respecto al Grupo Control.

\section{Discusión}

La angiogénesis se puede definir como la formación de vasos sanguíneos nuevos a partir de vasos preexistentes. En adultos el recambio de células endoteliales es bajo y la revascularización está generalmente asociada a procesos de cicatrización y regeneración de tejidos ${ }^{4,27}$. La cuantificación de la neovascularización ha sido utilizada en múltiples modelos en un afán de generar medidas terapéuticas.

Nos propusimos aplicar un modelo de estudio de angiogénesis utilizando la MAC dado su simplicidad, bajo costo de implementación y reproducibilidad. Uno de los objetivos fue aprender a utilizar el modelo de MAC para el estudio de la angiogénesis, el que resultó un modelo relativamente fácil de aplicar, pero con el problema de la pérdida de $20 \%$ de los huevos fecundados, que murieron durante el período de incubación. Por otro lado creemos que la MAC es un modelo que permite evaluar de manera in vivo la angiogénesis permitiendo observar de manera macroscópica y microscópica los efectos de la intervención experimental a analizar.

Respecto al uso de un inhibidor de PDE se puede decir que existen actualmente escasos reportes del uso de estos para modificar la angiogénesis, la mayoría de ellos han demostrado su rol angiogénico como extrapolación de los resultados obtenidos ${ }^{14,18}$.

En relación con los resultados obtenidos podemos decir que estos fueron concordantes con nuestra hipótesis, demostrándose un efecto angiogénico claro del SDF incluso a dosis bajas, lo que en otros estudios no había sido demostrado. De hecho, incluso con una dosis de SDF de $1 \mathrm{mg} / \mathrm{kg}$ se generó una mayor angiogénesis, en relación al control, de manera significativa, lo que permite suponer que incluso con dosis menores posiblemente se mantendría dicho efecto. Además, pudimos demostrar que con dosis crecientes de SDF, el efecto angiogénico también se hacía más intenso, no evidenciándose muertes de individuos con dosis elevadas de $10 \mathrm{mg} / \mathrm{kg}$, dosis donde se generó casi el doble de la angiogénesis en comparación con el control. Finalmente podemos decir que el SDF demostró que es proangiogénico en todas las dosis utilizadas, lo que nos permite asegurar que el SDF es una sustancia proangiogénica potente.

$\mathrm{Al}$ analizar la CAM del SDF, utilizamos un modelo de regresión lineal donde nuestro fin fue determinar la dosis y/o concentración de SDF requerida, para generar una angiogénesis significativamente mayor a la generada por la angiogénesis inflamatoria provocada por el disco de metilcelulosa. Respecto a los resultados de dicha regresión lineal, podemos decir que fue posible aplicarla dada la relación lineal que presentaba el SDF en relación al aumento del número de vasos de la $\mathrm{MAC}$. $\mathrm{Al}$ analizar la regresión lineal de nuestro experimento pudimos determinar que la CAM para el SDF es de $0,24 \mathrm{mg} / \mathrm{kg}$ o 0,16 ug/uL.

La angiogénesis es un proceso complejo que puede ser modificado o intervenido de múltiples maneras. Respecto a la angiogénesis, existe acuerdo que el SDF permitiría un aumento del cGMP intracelular la cual activaría la vía del VEGF por medio de la activación de las PKA ${ }^{17}$. 


\section{Referencias}

1. Pluda J. Tumor associated angiogénesis: mechanisms, clinical implications and therapeutics strategies. Semin Oncol. 1997;24:203-8.

2. Malonne H, Langer I, Kiss R, Atassi G. Mechanisms of tumor angiogénesis and therapeutics implications: angiogenic inhibitors. Clinical \& Exp Metast. 1999;17:114.

3. Danekamp J. Vascular attack as a therapeutic strategy for cancer. Cancer Metast Rev. 1990;3:267.

4. Folkman J. How is blood vessel growth regulated in normal and neoplastic tissue? Cancer Res. 1986;46:467.

5. Bostwick D, Iczkowski K. Microvessel density in prostate cancer: prognostic and therapeutic utility. Sem in Urol Oncology 1998;16:118-3.

6. Andrades P, Sepulveda S. Universidad de Chile (eds.). Cirugía Plástica Esencial 2005, pp 45-65.

7. Maragoudakis ME. Angiogenesis. Yakoub, M. \& Pepper J. (eds.). Annual of Cardiac Surgery 1993, pp $13 \pm 19$. London: Current Science.

8. Cheitlin MD, Hutter AM, Brindis RG, Ganz P, Kaul S, Russell RO, et al. Use of sildenafil (Viagra) in patients with cardiovascular disease. Circulation 1999;99:16877.

9. Borlaug BA, Melenovsky V, Marhin T, Fitzgerald P, Kass DA. Sildenafil inhibits beta-adrenergic-stimulated cardiac contractility in humans. Circulation. 2005; 112:2642-9.

10. Das S, Maulik N, Das DK, Kadowitz PJ, Bivalacqua TJ. Cardioprotection with sildenafil, a selective inhibitor of cyclic 3',5'-monophosphate-specific phosphodiesterase 5. Drugs Exp Clin Res. 2002;28:213-9.

11. Ziche M, Morbidelli L, Masini E, Granger HJ, Geppet TI, Ledda F. Nitric oxide promotes DNA síntesis and cyclic GMP formation in endothelial cells from postcapillary venules. Biochem Biophys Res Común. 1997;192:1198-203.

12. Gallo O, Masini E, Morbidelli L, Franchi A, FiniStorchi I, Vergari WA, et al. Role of nitric oxide in angiogenesis and tumor progression in head and neck cancer. J. Natl Cancer Inst. 1998;90;587-96.

13. Parenti A, Morbidelli L, Cui XL, Douglas JC, Hood JD, Granger HJ, et al. Nitric oxide is an upstream signal of vascular endothelial growth factor induced extracellular signal regulated kinase $1 / 2$ activation in post capillary endothelium. J Biol Chem. 1998;273: 4220-6.

14. Pyriochou A, Zhou Z, Koika V, Petrou C, Cordopatis P, Sessa WC, et al. The phosphodiesterase 5 inhibitor sildenafil stimulates angiogenesis through a protein kinase G/MAPK pathway. J Cell Physiol. 2007;211:197-204.
15. Breier G. Angiogenesis in embryonic development-A Review. Placenta 21, suplement A, Trophoblast Research 2000;14:S11-5.

16. Zhang R, Wang L, Zhang L, Chen J, Zhu Z, Zhang Z, et al. Nitric oxide enhances angiogenesis via the synthesis of vascular endothelial growth factor and cGMP after stroke in the rat. Circ Res. 2003;92:308-13.

17. Jozkowicz A, Cooke JP, Guevara I, Huk I, Funovics P, Pachinger $\mathrm{O}$, et al. Genetic augmentation of nitric oxide synthesis increases the vascular generation of VEGF. Cardiovasc Res. 2001;51:773-83.

18. Dulak J, Jozkowicz A, Dembinska-Kiec A, Guevara I, Zdzienicka A, Zmudzinska-Grochot D, et al. Nitric oxide induces the synthase of vascular endothelial growth factor by rat vascular smooth muscle cells. Arterioscler Thromb Vasc Biol. 2000;20:659-66.

19. Sadhu K, Hensley K, Florio VA, Wolda SL. Differential expression of the cyclic GMP-stimulated phosphodiesterase PDE2A in human venous and capillary endotelial cells. J Histochem Cytochem. 1999;47:895-906.

20. Favot L, Keravis T, Holl V, Le Bec A, Lugnier C. VEGF-induced HUVEC migration and proliferation are decreased by PDE2 and PDE4 inhibitors. Thromb Haemost. 2003;90:334-43.

21. Thompson WJ, Ashikaga T, Kelly JJ, Liu L, Zhu B, Vemavarapu L, et al. Regulation of cyclic AMP in rat pulmonary microvascular endothelial cells by rolipramsensitive cyclic AMP phosphodiesterase (PDE4). Biochem Pharmacol. 2002;63:797-807.

22. Papapetropoulos A, García-Cardena G, Madri JA, Sessa WC. Nitric oxide production contributes to the angiogenic properties of vascular endothelial growth factor in human endothelial cells. J Clin Invest. 1997;100:313139.

23. Fukumura D, Gohongi T, Kadambi A, Izumi Y, Ang J, Yun C-O, et al. Predominant role of endothelial nitric oxide synthase in vascular endothelial growth factorinduced angiogenesis and vascular permeability. Proc Natl Acad Sci USA 2001;98:2604-9.

24. Lue TF. Erectile dysfunction. N Engl J Med. 2000;342: 1802-13.

25. Rabe KF, Tenor H, Dent G, Schudt C, Nakashima M, Magnussen H. Identification of PDE isozymes in human pulmonary artery and effect of selective PDE inhibitors. Am J Physiol. 1994;266:L536-43.

26. Boolell M, Allen MJ, Ballard SA. Sildenafil: an orally active type 5 cyclic GMP-specific phosphodiesterase inhibitor for the treatment of penile erectile dysfunction. Int J Impot Res. 1996;8:47-52.

27. Carmeliet P. Angiogenesis in health and disease. Nat Med. 2003;9:653-60. 